\begin{tabular}{|c|c|c|c|c|c|c|c|c|c|c|c|c|c|c|c|c|c|c|c|c|c|c|}
\hline \multicolumn{2}{|c|}{1903} & \multicolumn{3}{|c|}{ M.7. Leipz. } & \multirow{2}{*}{\multicolumn{2}{|c|}{$\begin{array}{c}\Delta \alpha \\
-o^{m} 34.28\end{array}$}} & \multicolumn{3}{|c|}{$\Delta d$} & \multirow{2}{*}{$\begin{array}{c}\mathrm{Vgl} \\
54\end{array}$} & \multirow{2}{*}{$\begin{array}{c}\mathrm{Bb} \\
-\cdots \\
\mathrm{F}\end{array}$} & \multicolumn{3}{|c|}{$\alpha$ app } & $\log p \cdot \Delta_{i}$ & \multicolumn{3}{|c|}{$\delta$ app. } & \multirow{2}{*}{$\left.\right|_{-} ^{\mid}$} & \multicolumn{3}{|c|}{ Red. ad l. app. * * } \\
\hline ni & 27 & 13 & & 41.2 & & & & & & & & & 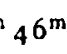 & 415.26 & & & - & & & 8 & & \\
\hline & 27 & 13 & 38 & 46.9 & & - & - & $0^{\prime} 4$ & $47: 3$ & 10 & $\mathbf{F}$ & & - & & & $-2^{\circ}$ & & 39.4 & 0.849 & - & +16.1 & \\
\hline & 27 & 13 & 54 & 2.8 & & - & + & $\circ$ & 2.5 & 10 & F & & - & & & 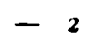 & 9 & & 850 & - & $+16.1=$ & \\
\hline & 28 & 11 & 40 & 27.6 & -0 & 0.63 & & - & & 39 & F & 21 & 15 & 8 & $482 n$ & & - & & - & -2.62 & - & \\
\hline & 28 & 12 & 2 & 28.1 & & - & + & 4 & 55.4 & 15 & $\mathrm{~F}$ & & - & & 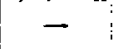 & $-\circ$ & $5^{8}$ & & 0. & - & & \\
\hline \multirow[t]{10}{*}{ uli } & II & 9 & 50 & $3 \cdot 3$ & -0 & 28.31 & + & 95 & 59.2 & 12.4 & $\mathrm{H}$ & 2 & 49 & 8 & & +33 & 14 & & 9 & 1 & & \\
\hline & I 1 & 10 & 0 & 2.4 & -0 & $20 i$ & + & 23 & 31.1 & 10.4 & $\mathrm{H}$ & 20 & 49 & 74 & $9 \cdot 5$ & +33 & 16 & 14.2 & 7 & 3 & $6 \vdots$ & \\
\hline & 13 & 10 & I & 46.3 & +0 & 25 & + & 3 & 2.3 & 12.4 & $\mathrm{H}$ & 20 & 24 & 22.00 & $9 \cdot 5$ & 42 & 52 & $5^{2.3}$ & 6 & 7 & & \\
\hline & 13 & 10 & 11 & 32.8 & +0 & 48.37 & + & 0 & I 3.1 & 12.4 & $\mathrm{H}$ & 20 & 24 & 15.82 & & 42 & 54 & 1 & & & & \\
\hline & 14 & 9 & 32 & 24.4 & +1 & 29.54 & - & 6 & 39.1 & 12.4 & $\mathrm{H}$ & 2 & 8 & 38 & & 47 & 47 & 6.6 & 267 & 5 & 4 & \\
\hline & 16 & 9 & $5^{6}$ & 28.7 & -0 & 3.81 & & - & & 12 & $\mathrm{~F}$ & 19 & 18 & 28.90 & $66_{n}$ & & - & & - & -3.51 & 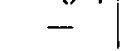 & \\
\hline & 16 & 10 & 4 & 29.8 & & - & + & 8 & 17.1 & 1 & $\mathbf{F}$ & & - & & & +57 & $3^{6}$ & 14.1 & $9.744 n$ & - & +15.3 & \\
\hline & 16 & 10 & 26 & 14.9 & 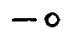 & 41.95 & & - & & 3 & F & 19 & 17 & 50.76 & $9.35^{8} n$ & & - & & - & +3.51 & - & \\
\hline & 16 & 10 & 30 & 12.7 & & - & +1 & & 3.0 & I & $\mathbf{F}$ & & - & & - & +57 & 41 & 0.0 & $9.880_{n}$ & - & +15.3 & \\
\hline & 26 & 9 & 15 & 22.7 & +3 & $3^{6}$ & +1 & & 59.4 & 12.4 & $\mathrm{H}$ & 12 & 48 & 26 & & $+6 i$ & 3 & 2.8 & 0.228 & $-c$ & + & \\
\hline \multirow[t]{5}{*}{ aug. } & 6 & 8 & $5^{8}$ & 53.1 & +2 & & - & I & 46.1 & $9 \cdot 3$ & $\mathrm{H}$ & 1 & 19 & 27.27 & & +45 & $5^{\circ}$ & 1.9 & & -0.16 & -2.4 & \\
\hline & 7 & 8 & $4 !$ & 21.4 & -2 & 8.16 & - & 2 & 31.0 & 12.4 & $\mathrm{H}$ & 1 & 15 & 31.61 & 9.7 & +44 & 52 & $5 \cdot 5$ & 0.723 & +0.19 & $-\quad 2.6$ & \\
\hline & 7 & 8 & 58 & 3.8 & -1 & 57.34 & + & 0 & 23.1 & 124 & $\mathrm{H}$ & 11 & 15 & 29.28 & 9.7 & +44 & $5^{3}$ & 25.5 & 0.748 & +0.19 & -2.6 & \\
\hline & 8 & 8 & 49 & 53.2 & +2 & 24.13 & + & 3 & 45.0 & 12.4 & $\mathrm{H}$ & I & 11 & 45.26 & & +43 & 55 & 23.0 & & +0.22 & -3.4 & \\
\hline & 8 & 8 & 49 & 53.2 & -3 & 27.22 & - & 0 & 20.1 & 12.4 & $\mathrm{H}$ & 11 & II & $45 \cdot 42$ & 0. & +43 & 55 & 9.4 & & +0.23 & 3.0 & \\
\hline
\end{tabular}

Bemerkung zu Juli 16 und 26: I, uft sehr dunstig, schwierige Beobachtung.

Mittlere Örter der Vergleichsterne.

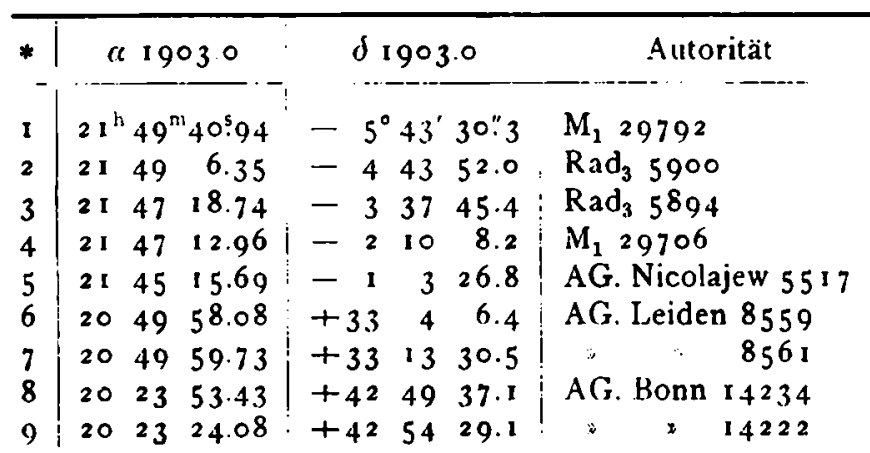

Leipzig, 1903 Nov. 27.

\begin{tabular}{|c|c|c|c|c|c|c|c|c|c|c|}
\hline * & \multicolumn{3}{|c|}{ \& 1903.0} & \multicolumn{3}{|c|}{$d 1903.0$} & \multicolumn{4}{|c|}{ Autorität } \\
\hline 10 & $20^{h}$ & 7 & $n_{1}: 39$ & +47 & ${ }^{\circ} 53$ & $32 " 3$ & $A G$. & Bonn & $13^{8} 53$ & \\
\hline 11 & 19 & 18 & 29.20 & +57 & 27 & 41.7 & $\mathbf{A G}$ & Helsin & ngfors 1 & 10399 \\
\hline 12 & 12 & 44 & 25.91 & +60 & 50 & 55.7 & $>$ & - & - & 7318 \\
\hline 13 & I I & 16 & 30.00 & +45 & $5 \pi$ & 50.4 & AG. & Bonn & 8000 & \\
\hline 14 & II & 17 & $39.5^{8}$ & +44 & 54 & 39.1 & メ & \% & 8010 & \\
\hline 15 & 11 & 17 & 26.43 & +44 & 51 & 5.0 & : & $\because$ & 8007 & \\
\hline 16 & 11 & 9 & $20.9 \mathrm{t}$ & +43 & 51 & 41.4 & $\because$ & i & 7943 & \\
\hline 17 & II & 15 & 12.41 & +43 & 55 & 42.5 & $\because$ & , & 7992 & \\
\hline
\end{tabular}

I. Hayn.

Observations de la comète $1903 \mathrm{IV}$

faites au réfracteur $(245 \mathrm{~mm})$ de l'Observatoire de Kiew.

\begin{tabular}{|c|c|c|c|c|c|c|c|c|c|c|c|c|c|c|c|c|c|c|c|c|}
\hline \multirow{2}{*}{\multicolumn{2}{|c|}{$\frac{1903}{\text { Juill. } 14}$}} & \multicolumn{3}{|c|}{ T. m. Kiew } & \multicolumn{3}{|c|}{$\Delta \alpha$} & & \multicolumn{3}{|c|}{$\Delta d$} & \multicolumn{3}{|c|}{$\alpha$ app. } & \multicolumn{3}{|c|}{$\delta$ app. } & \multicolumn{2}{|c|}{ Red. ad 1. app. } & * \\
\hline & & 10 & 59 & $n_{41}$ & + & & $45^{5}: 05$ & - & & $34 " 7$ & I & $20^{h}$ & $7^{m}$ & $23^{5} .14$ & $+47^{\circ}$ & $5^{\circ}$ & $51 " 4$ & $+3 \cdot 46$ & +13.4 & I \\
\hline & 14 & I I & 6 & 30 & - & 0 & 22.84 & - & 3 & 42.6 & 5 & 20 & 7 & 18.18 & +47 & 53 & 6.4 & +3.46 & +13.4 & 2 \\
\hline & 15 & 10 & 5 & 20 & - & $\mathbf{I}$ & 27.90 & - & 5 & 15.8 & 7 & 19 & $4^{6}$ & $47 \cdot 39$ & +52 & 39 & 30.6 & $+3 \cdot 5^{2}$ & +14.1 & 3 \\
\hline & 16 & 10 & 5 & 21 & + & I & $15.2 \mathrm{I}$ & + & 5 & 6.5 & 5 & & - & & & - & & - & - & 4 \\
\hline & 17 & 10 & 14 & & + & $\mathbf{I}$ & 43.83 & + & 4 & 30.5 & 8 & 18 & 45 & 28.63 & $+6 \mathrm{I}$ & $3^{6}$ & 26.4 & +3.39 & +16.8 & 5 \\
\hline & 18 & 10 & 21 & 12 & & - & - & & - & & - & 18 & 3 & 2.90 & +65 & 2 & 20.2 & +2.79 & +14.5 & - \\
\hline & 19 & 9 & 30 & 33 & & - & - & & - & & - & 17 & 15 & 11.55 & +67 & 19 & 51.9 & +2.53 & +18.9 & - \\
\hline & 21 & 10 & I & 14 & + & 2 & 47.97 & + & 5 & 2.0 & 7 & 15 & 27 & 33.05 & +68 & 39 & 11.9 & +0.87 & +17.8 & 6 \\
\hline & 22 & II & I & 59 & - & I & 3.42 & + & 5 & 46.6 & 7 & & - & - & & - & & - & - & 7 \\
\hline & 26 & 11 & 8 & 24 & + & 3 & $5 \cdot 55$ & + & 8 & 37.9 & 10 & 12 & 47 & 31.11 & +60 & 59 & 41.7 & -0.34 & +7.6 & 8 \\
\hline & 27 & 9 & 51 & $4^{8}$ & + & 7 & 10.75 & - & $\mathbf{I}$ & 12.5 & 8 & 12 & 32 & 42.29 & +59 & 17 & 9.9 & -0.20 & +6.5 & 9 \\
\hline & 28 & 10 & 16 & 9 & + & 8 & 54.01 & - & 2 & $5^{8.1}$ & 5 & 12 & & 31.51 & +57 & $3 \mathbf{I}$ & 23.5 & -0.22 & +5.1 & 10 \\
\hline
\end{tabular}




\begin{tabular}{|c|c|c|c|c|c|c|c|c|c|c|c|c|c|c|c|c|c|c|}
\hline \multicolumn{2}{|c|}{1903} & \multicolumn{3}{|c|}{ T. m. Kiew } & \multicolumn{2}{|r|}{$\Delta \alpha$} & \multicolumn{2}{|r|}{$\Delta \delta$} & Cp. & \multicolumn{2}{|c|}{$\alpha$ app. } & \multicolumn{3}{|c|}{$\delta$ app. } & \multicolumn{4}{|c|}{ Red. ad 1. app. |* } \\
\hline iill. & 28 & $10^{h}$ & $5^{6^{m}}$ & ${ }^{n} 47^{9}$ & -1 & $m_{1} 3^{s} .05$ & +9 & $9^{\prime} 14: 6$ & 5 & $12^{\mathrm{h}} 1$ & 11.85 & $+57^{\circ}$ & $28^{\prime}$ & 16."2 & -0.22 & +5 & 5.1 & I \\
\hline & $3^{\circ}$ & 10 & 54 & 5 & +10 & 36.77 & +3 & 353.5 & 5 & 115 & 20.52 & +54 & 17 & 58.2 & -0.12 & +2 & 2.9 & 12 \\
\hline Loût & 5 & 9 & 4 & 2 & & 32 & +4 & 34.0 & 10 & I 2 & 53 & +46 & 54 & 18.4 & +0.13 & -1 & 1.6 & 13 \\
\hline & 7 & 9 & 35 & 22 & +11 & 22.06 & -8 & 8350 & 6 & II 1 & 35.04 & +44 & 52 & 50.5 & +0.19 & -2 & $2 \cdot 7$ & 14 \\
\hline & 8 & 10 & 51 & $5^{8}$ & -5 & 52.20 & -7 & 732.8 & 8 & 111 & 38.51 & +43 & 53 & I 8.6 & +0.22 & -3 & $3 \cdot 3$ & 15 \\
\hline & 12 & 9 & $5^{6}$ & 20 & -2 & 31.04 & +5 & $5 \quad 52.2$ & 10 & 105 & 12.23 & +40 & 24 & 56.1 & +0.33 & -5 & 5.1 & 10 \\
\hline & 14 & 9 & 9 & 55 & -7 & $9 \cdot 36$ & -0 & 27.6 & 7 & 105 & $5^{8.84}$ & +38 & 45 & 17.5 & $+0.3^{8}$ & -6 & 6.0 & 17 \\
\hline & 15 & 9 & 0 & 32 & +6 & 0.48 & -10 & 4.3 & 6 & 104 & 54.18 & +37 & 54 & & +0.40 & -6 & 6.4 & 18 \\
\hline & 18 & 8 & 34 & 58 & +4 & 5.16 & +5 & $5 \quad 47.2$ & 10 & 103 & 46.43 & +35 & 17 & 18.9 & +0.44 & -8 & 8.8 & 19 \\
\hline
\end{tabular}

Juill. 18 et 19 . Comète observée au cercle méridien. L'AR. du 19 juillet fut corrigée de $-1^{\mathrm{m}}$.

Positions moyenues des étoiles de comparaison.

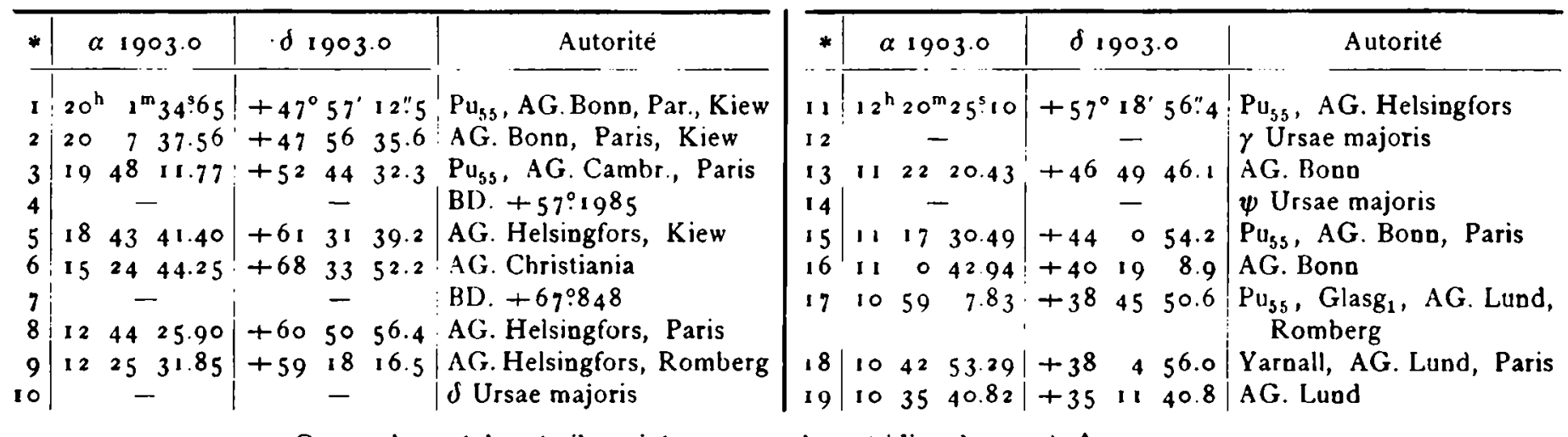

** 1, 2, 5. On a observé les étoiles citées au cercle méridien le 27 Août r 903.

Kiew, 1903 Novembre 14.

M. Ditchenko, Astronome-Observateur.

\section{Observations de la comète 1903 IV}

faites à l'Observatoire d'Alger à l'équatorial coudé de $0.318 \mathrm{~m}$ d'ouverture par MM. Rambaud, Sy et Villatte.

\begin{tabular}{|c|c|c|c|c|c|c|c|c|c|c|c|c|c|c|c|c|c|c|c|c|}
\hline \multirow{2}{*}{\multicolumn{2}{|c|}{$\frac{1903}{\text { Juill. } 25}$}} & \multicolumn{3}{|c|}{ T. m. Alger } & \multicolumn{2}{|c|}{$\Delta \alpha$} & \multicolumn{2}{|c|}{$\Delta \delta$} & \multirow{2}{*}{$\begin{array}{l}\text { Cp. } \\
12.12\end{array}$} & \multicolumn{3}{|c|}{$\alpha$ app. } & $\log p . \Delta$ & \multicolumn{3}{|c|}{$\delta$ app. } & \multirow{2}{*}{$\frac{\log p \cdot \Lambda}{9.881_{\mathrm{n}}}$} & \multicolumn{2}{|c|}{ Red. ad 1. app. } & \multirow[b]{2}{*}{ I } \\
\hline & & & 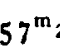 & $23^{8}$ & 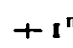 & 4.01 & +8 & $4: 9$ & & $13^{h}$ & & .02 & 9.953 & $+62^{\circ}$ & $53^{\prime}$ & 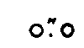 & & $-0: 31$ & 8 & \\
\hline & 25 & 9 & 19 & 42 & 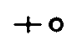 & $45 \cdot 35$ & +6 & 24.6 & 12.16 & 13 & 6 & & 9.975 & +62 & 51 & & $9 \cdot 304 n$ & -0.31 & & \\
\hline & 27 & 9 & 33 & 33 & & & +14 & 31.7 & 2.12 & 12 & 31 & & & +59 & 10 & & j & & & \\
\hline & 27 & 9 & 59 & 27 & & & +12 & 3 & 2.12 & 12 & 31 & & & +59 & 8 & 5 & & & & \\
\hline & 28 & 9 & 59 & 37 & -1 & & +5 & 48.1 & 2.16 & 12 & 18 & & 9.8 & +57 & 24 & 49 & & & & \\
\hline & 28 & 103 & 30 & 2 & $-I$ & & +3 & 38.2 & 2.16 & 12 & 18 & & & +57 & 22 & & 69 & 0 & & \\
\hline & 29 & 9 & 53 & 19 & -2 & & -15 & $5 I$ & 2. J 2 & 12 & 8 & & & +55 & 47 & & & & & \\
\hline & 29 & & 2 & 55 & -2 & 23 & -16 & 3 & 2 & 12 & 8 & 4.89 & & +55 & 46 & & & & & \\
\hline & 30 & 101 & 16 & 47 & $+I$ & $5^{8}$ & -13 & 23.8 & 0 & 11 & $5^{8}$ & 56.22 & & +54 & 3 & 32 & 30 & & .7 & \\
\hline & I & 103 & 39 & 21 & +2 & & +5 & 20.1 & 18.12 & 11 & 44 & 65 & & +51 & 24 & & 38 & & & \\
\hline & 3 & 91 & 173 & $3^{2}$ & & & +1 & 26. & 12.12 & 11 & 33 & & & +49 & 1 & & & & & \\
\hline & 3 & 94 & 413 & $3^{\circ}$ & + & & & 1 & & 11 & $3^{2}$ & & & +48 & 59 & 8.7 & 0. & & & \\
\hline & 4 & 10 & 2 & 10 & + & 53 & +4 & & & 11 & 27 & & & +47 & 51 & & & & & \\
\hline & 5 & & 9 & 28 & $-I$ & & +1 & 50 & I 2.16 & II & 23 & & & +46 & 49 & & & & -1.5 & \\
\hline & 5 & 93 & 32 & 31 & -1 & 12. & +0 & 46. & & t t & 23 & 34 & & +46 & 48 & & 0.698 & & -1.5 & \\
\hline & 8 & & 3 & 45 & +0 & 14.8 & +4 & 55. & 12.14 & I I & I I & 41.28 & & +43 & 53 & & & & & \\
\hline & 8 & 92 & 20 & 27 & +0 & 12.06 & +4 & 24.7 & 12.12 & 11 & I I & $3^{8 .} 5^{1}$ & & +43 & 52 & $4^{6} 6.3$ & 0.729 & +0.23 & $-3 \cdot 3$ & \\
\hline
\end{tabular}

